\title{
CORRECTION
}

\section{Correction: Transitions: navigating career choices throughout the lifespan}

Philip Pizzo and Norman D. Rosenblum

Pediatric Research (2022) 91:1918; https://doi.org/10.1038/s41390-020-01214-7

Correction to: Pediatric Research https://doi.org/10.1038/s41390020-01127-5, published online 24 September 2020

The original version of this Article omitted the middle initial of the author Norman D. Rosenblum.
This has now been corrected in both the PDF and HTML versions of the Article. 